\title{
The uses of Connes and Kreimer's algebraic formulation of renormalization theory
}

\author{
Héctor Figueroa* and José M. Gracia-Bondía $\nmid$ † \\ * Department of Mathematics, \\ $\dagger$ Department of Physics, \\ Universidad de Costa Rica, 2060 San Pedro, Costa Rica \\ and \\ $\ddagger$ Department of Theoretical Physics, Universidad de Zaragoza, \\ 50009 Zaragoza, Spain
}

November 17, 2018

\begin{abstract}
We show how, modulo the distinction between the antipode and the "twisted" or "renormalized" antipode, Connes and Kreimer's algebraic paradigm trivializes the proofs of equivalence of the (corrected) Dyson-Salam, Bogoliubov-Parasiuk-Hepp and Zimmermann procedures for renormalizing Feynman amplitudes. We discuss the outlook for a parallel simplification of computations in quantum field theory, stemming from the same algebraic approach.
\end{abstract}

Keywords: Feynman diagrams, renormalization, graded Hopf algebras, antipodes. PACS numbers: 11.10.Gh, 02.20.Uw

\section{Introduction}

The present authors have dealt in a Hopf algebraic context with the relation between the DysonSalam, Bogoliubov-Parasiuk and Zimmermann renormalization schemes in Ref. 1. This we did using the algebra of rooted trees $H_{R}$ (Ref. 2) as a proxy for the complexities of the combinatorics of Feynman graphs in renormalization.

The point of Ref. 1 was that the differences of the diverse schemes could largely be tracked down to avatars of the convolution operation in spaces of homomorphisms of Hopf algebra. This was illustrated by the apparently simple-minded, but tremendously effective, computation of antipode images in $H_{R}$ by means of the convolution geometric series. 
In this paper we return on the subject, now in terms of Hopf algebras of the Feynman graphs themselves.

It must be acknowledged that the deeply conceptual approach by Connes and Kreimer till now has failed to impress many physicists who practise the renormalization theory. For that approach to become mainstream, it should be shown to simplify both proofs and calculations in perturbative renormalization theory.

The proof given by Zimmermann ${ }^{3}$ of the equivalence of his forest formula and Bogoliubov and Parasiuk's scheme has a reputation of difficulty. The Dyson-Salam scheme has been thoroughly analyzed and purged of difficulties in Ref. 4. But the proof of equivalence between the forest and the (corrected) Dyson-Salam formulae given in Ref. 4 is anything but simple.

In contrast, here we show that the equivalence between all the aforementioned schemes boils down to downright basic facts in Hopf algebra theory. So basic in fact, that we can only conclude that the combinatorics of perturbative renormalization finds its definitive expression in Hopf algebraic terms. Not only the equivalence proofs result from uniqueness of the Hopf antipode, but we are able to show that the Dyson-Salam scheme corresponds identically to the convolution geometric series. All our arguments are elementary and short, much more so, by the way, than in Ref. 1: in the previous paper, we did not quite see the forest for the trees.

At the end of the paper we briefly review the perspectives for the Connes-Kreimer method to simplify other theorems and computations in renormalization, by means of reduction to the case of elements that are primitive (i.e., without subdivergences). Here the situation is more mixed; still, the hope remains that sizeable simplifications can be gleaned from the algebraic approach.

\section{Bialgebras of graphs}

The basics of graded bialgebra theory are recalled in the Appendix. From now on, we assume the reader is familiar with them.

Bialgebras of Feynman graphs, encoding the combinatorics of renormalization, were introduced by Connes and Kreimer in Ref. 5. The precise definition we use in this paper was first given in Ref. 6. To fix ideas, and whenever an example is given, we think of the (massless) $\varphi_{4}^{4}$ scalar model. Nevertheless, the constructions hold in any given quantum field theory, such as the $\varphi_{6}^{3}$ model considered in Ref. 5.

We recall that a graph or diagram $\Gamma$ of the theory is specified by a set $\mathcal{V}(\Gamma)$ of vertices and a set $\mathcal{L}(\Gamma)$ of lines (propagators) among them; external lines are attached to only one vertex each, internal lines to two. Diagrams with no external lines will not be taken into account —and in $\varphi_{4}^{4}$ theory only graphs with an even number of external lines are to be found. Also tadpole diagrams, in which a line connects a vertex to itself, are excluded in this paper.

Given a graph $\Gamma$, a subdiagram $\gamma$ of $\Gamma$ is specified by a subset of at least two elements of $\mathcal{V}(\Gamma)$ and a subset of the lines that join these vertices in $\Gamma$. By exception, the empty subset $\emptyset$ will be admitted as a subdiagram of $\Gamma$. As well as $\Gamma$ itself. Clearly, the external lines for a subdiagram $\gamma$ include not only a subset of original incident lines, but some internal lines of $\Gamma$ not included in $\gamma$. The connected pieces of $\Gamma$ are the maximal connected subdiagrams. A diagram is proper (or 1PI) when the number of its connected pieces would not increase on the removal of a single internal line; otherwise it is called improper. An improper graph is the union of proper components plus subdiagrams containing a single line. 
A subgraph of a proper graph is a subdiagram that contains all the elements of $\mathcal{L}(\Gamma)$ joining its vertices in the whole graph; as such, it is determined solely by the vertices. When a subdiagram contains several connected pieces, each one of them being a subgraph, we still call it a subgraph. A subgraph of an improper graph $\Gamma$, distinct from $\Gamma$ itself, is a proper subdiagram each of whose components is a subgraph with respect to the proper components of $\Gamma$.

We write $\gamma \subseteq \Gamma$ if and only if $\gamma$ is a subgraph of $\Gamma$ as defined (not just a subdiagram): this is the really important concept for us. For renormalization in configuration space, ${ }^{\dagger}$ it is more convenient to deal with subgraphs than with more general subdiagrams. Zimmermann showed long ago that only subtractions corresponding to subgraphs need be used, ${ }^{8}$ and this dispenses us from dealing with subdiagrams that are not subgraphs.

Two subgraphs $\gamma_{1}, \gamma_{2}$ of $\Gamma$ are said to be nonoverlapping when $\gamma_{1} \cap \gamma_{2}=\emptyset$ or $\gamma_{1} \subseteq \gamma_{2}$ or $\gamma_{2} \subseteq \gamma_{1}$; otherwise they are overlapping. Given $\gamma \subseteq \Gamma$, the quotient graph or cograph $\Gamma / \gamma$ (reduced graph in Zimmermann's parlance) is defined by shrinking $\gamma$ in $\Gamma$ to a point, that is to say, $\gamma$ (bereft of its external lines) is considered as a vertex of $\Gamma$, and all the lines in $\Gamma$ not belonging to $\gamma$ belong to $\Gamma / \gamma$. This is modified in the obvious way when $\gamma$ represents a propagator correction. The graphs $\Gamma$ and $\Gamma / \gamma$ have the same external structure. A nonempty $\Gamma / \gamma$ will be proper iff $\Gamma$ is proper - the situation considered in Ref. 5.

Now, the bialgebra $\mathcal{H}$ is defined as the polynomial algebra generated by the empty set $\emptyset$ and the connected Feynman graphs that are (superficially) divergent and/or have (superficially) divergent subgraphs (renormalization parts in Zimmermann's parlance), with set union as the product operation (hence $\emptyset$ is the unit element $1 \in \mathcal{H}$ ). The counit is given by $\varepsilon(\Gamma):=0$ on any generator, except $\varepsilon(\emptyset)=1$.

The really telling operation is the coproduct $\Delta: \mathcal{H} \rightarrow \mathcal{H} \otimes \mathcal{H}$; as it is to be a homomorphism of the algebra structure, we need only define it on connected diagrams. By definition, the coproduct of $\Gamma$ is given by

$$
\Delta \Gamma:=\Gamma \otimes 1+1 \otimes \Gamma+\sum_{\emptyset \subseteq \gamma \subsetneq \Gamma} \gamma \otimes \Gamma / \gamma=\sum_{\emptyset \subseteq \gamma \subseteq \Gamma} \gamma \otimes \Gamma / \gamma .
$$

The sum is over all divergent, proper, not necessarily connected subgraphs of $\Gamma$, such that each piece is divergent, including (and then with the possible exception of, as $\Gamma$ need not be divergent nor proper) the empty set and $\Gamma$ itself. We put $\Gamma / \Gamma=1$. When appropriate, the sum runs also over different types of local counterterms associated to $\gamma$ (see Refs. 5,9); this is not needed in our example model.

It is natural to exclude the appearance of tadpole parts in $\Gamma / \gamma$, and this we do hereafter. This tadpole-free condition was not used or remarked in Ref. 6, nor in Ref. 10, which employs the same definition. We show in Figure 1 how this situation can happen. The cograph corresponding to the "bikini" subgraph in the upper part of the graph in Figure 1 is a tadpole correction and can be outlawed from the coproduct.

For the proof of the bialgebra properties of $\mathcal{H}$, we refer to Ref. 11; for graphical examples of coproducts, see Ref. 6.

Actually $\mathcal{H}$ is a connected, graded bialgebra. Obvious grading operators are available: if $\#(\Gamma)$ denotes the number of vertices in $\Gamma$ (i.e., the coupling order), then we define the degree of a generator (connected element) $\Gamma$ as $\nu(\Gamma):=\#(\Gamma)-1$; the degree of a product is the sum of the degrees of the factors. This grading is compatible with the coproduct, and clearly scalars are 


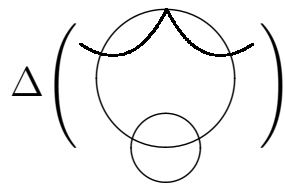

would contain the term
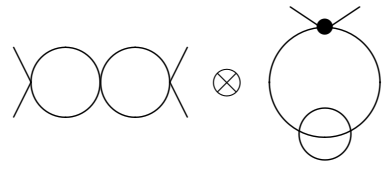

Figure 1: Cograph which is a tadpole part

the only degree 0 elements. Other gradings are by the number $I(\Gamma)$ of internal lines in $\Gamma$ and by loop number $\ell(\Gamma):=I(\Gamma)-\nu(\Gamma)$. For the $\varphi_{4}^{4}$ model, $\ell(\Gamma)=\nu(\Gamma)+1$ for two-point graphs and $\ell(\Gamma)=\nu(\Gamma)$ for four-point graphs. A more relevant grading will emerge in the next section.

Lurking in the background there is a character (i.e., multiplicative) map $f$ (the "Feynman rule") of $\mathcal{H}$ into an algebra $V$ of Feynman amplitudes: for instance, in dimensional regularization the character takes values in a ring of Laurent series (with finite order poles) in the regularization parameter. In physics, the Feynman rules are essentially fixed by the interpretation of the theory, and thus one tends to identify $\Gamma$ with $f(\Gamma)$.

\section{The importance of convolution}

Given a unital algebra $(A, m, u)$ and a counital coalgebra $(C, \Delta, \varepsilon)$ over $\mathbb{F}$, the convolution of two elements $f, g$ of the vector space of $\mathbb{F}$-linear maps $\operatorname{Hom}(C, A)$ is defined as the map $f * g \in$ $\operatorname{Hom}(C, A)$ given by the composition

$$
C \stackrel{\Delta}{\longrightarrow} C \otimes C \stackrel{f \otimes g}{\longrightarrow} A \otimes A \stackrel{m}{\longrightarrow} A .
$$

In other words, $f * g=m(f \otimes g) \Delta$. This product turns $\operatorname{Hom}(C, A)$ into a unital algebra, where the unit is the map $u \varepsilon$, as is easily checked. In particular, linear endomorphisms of a bialgebra can be convolved.

A bialgebra $H$ in which the identity map id $\operatorname{in}_{H}$ is invertible under convolution is called a Hopf algebra, and its convolution inverse $S$ is called the coinverse or antipode; that is to say, $\operatorname{id}_{H} * S=$ $S * \operatorname{id}_{H}=u \varepsilon$. The antipode is clearly unique. It is known to be of order two for commutative bialgebras. Also, in a commutative bialgebra $S$ is a homomorphism: $S(a b)=S(a) S(b)$.

In particular, if $\Delta(a)=\sum_{j} a_{j}^{\prime} \otimes a_{j}^{\prime \prime}$, then

$$
\varepsilon(a) 1_{H}=u \varepsilon(a)=m(S \otimes \mathrm{id}) \Delta(a)=\sum_{j} S\left(a_{j}^{\prime}\right) a_{j}^{\prime \prime},
$$

and likewise $\varepsilon(a) 1_{H}=\sum_{j} a_{j}^{\prime} S\left(a_{j}^{\prime \prime}\right)$. Since any left inverse under convolution automatically equals any right inverse provided both exist, any map $S$ satisfying (2) is the antipode.

The main outcome of equation (14) in the Appendix for connected graded bialgebras is that these are always Hopf. Indeed, as is done in Ref. 1, one can try to compute the antipode $S: H \rightarrow H$ by exploiting its very definition as the convolution inverse of the identity in $H$, via a geometric series:

$$
S:=(\mathrm{id})^{*-1}=(u \varepsilon-(u \varepsilon-\mathrm{id}))^{*-1}=u \varepsilon+(u \varepsilon-\mathrm{id})+(u \varepsilon-\mathrm{id})^{* 2}+\cdots
$$


Proposition 1. Let $H$ be a connected, graded bialgebra, then the geometric series expansion of $S(a)$ has at most $n+1$ terms when $a \in H^{(n)}$.

Proof. If $a \in H^{(0)}$ the claim holds since $(u \varepsilon-\mathrm{id}) 1=0$. Assume that the claim holds for the elements of $H^{(k)}$ when $k \leq n-1$, and let $a \in H^{(n)}$; then by (14)

$$
\begin{aligned}
(u \varepsilon-\mathrm{id})^{*(n+1)}(a) & =(u \varepsilon-\mathrm{id}) *(u \varepsilon-\mathrm{id})^{* n}(a) \\
& =m\left[(u \varepsilon-\mathrm{id}) \otimes(u \varepsilon-\mathrm{id})^{* n}\right] \Delta(a) \\
& =m\left[(u \varepsilon-\mathrm{id}) \otimes(u \varepsilon-\mathrm{id})^{* n}\right]\left(a \otimes 1+1 \otimes a+\Delta^{\prime} a\right) .
\end{aligned}
$$

The first two terms vanish because $(u \varepsilon-\mathrm{id}) 1=0$. By the induction hypothesis each of the summands of the third term are also zero.

As a corollary, connected graded bialgebras are always Hopf, with antipode indeed given by the geometric series (3). One of the advantages of this formulation is that we obtain fully explicit formulae for $S$ from the coproduct.

Proposition 2. If $a \in H^{(n)}, \Delta^{\prime}(a)=\sum_{j_{1}} a_{j_{1}}^{\prime} \otimes a_{j_{1}}^{\prime \prime}, \Delta^{\prime}\left(a_{j_{1}}^{\prime}\right)=\sum_{j_{2}} a_{j_{1} j_{2}}^{\prime} \otimes a_{j_{1} j_{2}}^{\prime \prime}$, and in general $\Delta^{\prime}\left(a_{j_{1}, \ldots, j_{k}}^{\prime}\right)=\sum_{j_{k+1}} a_{j_{1}, \ldots, j_{k+1}}^{\prime} \otimes a_{j_{1}, \ldots, j_{k+1}}^{\prime \prime}$, then for $1 \leq k \leq n-1$,

$$
(u \varepsilon-\mathrm{id})^{* k+1}(a)=(-1)^{k+1} \sum_{j_{1}, \ldots, j_{k}} a_{j_{1}, \ldots, j_{k}}^{\prime} a_{j_{1}, \ldots, j_{k}}^{\prime \prime} \cdots a_{j_{1} j_{2}}^{\prime \prime} a_{j_{1}}^{\prime \prime} .
$$

Proof. To abbreviate we use the notation $\sigma:=u \varepsilon-$ id. Then $\sigma(a)=-a$ if $a \in H^{(n)}$ with $n \geq 1$, because then $\varepsilon(a)=0$. Moreover,

$$
\begin{aligned}
\sigma^{* 2}(a) & =m(\sigma \otimes \sigma)\left(a \otimes 1+1 \otimes a+\Delta^{\prime} a\right) \\
& =\sum_{j_{1}} \sigma\left(a_{j_{1}}^{\prime}\right) \sigma\left(a_{j_{1}}^{\prime \prime}\right)=\sum_{j_{1}} a_{j_{1}}^{\prime} a_{j_{1}}^{\prime \prime},
\end{aligned}
$$

so the statement holds for $k=1$. If the statement holds for $\sigma^{* k}$, then

$$
\begin{aligned}
\sigma^{* k+1}(a) & =m\left(\sigma^{* k} \otimes \sigma\right)\left(a \otimes 1+1 \otimes a+\Delta^{\prime} a\right) \\
& =\sum_{j_{1}} \sigma^{* k}\left(a_{j_{1}}^{\prime}\right) \sigma\left(a_{j_{1}}^{\prime \prime}\right)=-\sum_{j_{1}} \sigma^{* k}\left(a_{j_{1}}^{\prime}\right) a_{j_{1}}^{\prime \prime} \\
& =(-1)^{k+1} \sum_{j_{1}, \ldots, j_{k}} a_{j_{1}, \ldots, j_{k}}^{\prime} a_{j_{1}, \ldots, j_{k}}^{\prime \prime} \cdots a_{j_{1} j_{2}}^{\prime \prime} a_{j_{1}}^{\prime \prime},
\end{aligned}
$$

since, by the induction hypothesis, $\sigma^{* k}\left(a_{j_{1}}^{\prime}\right)=(-1)^{k} \sum_{j_{2}, \ldots, j_{k}} a_{j_{1}, j_{2}, \ldots, j_{k}}^{\prime} a_{j_{1}, j_{2}, \ldots, j_{k}}^{\prime \prime} \cdots a_{j_{1}, j_{2}}^{\prime \prime}$.

If $\Delta_{i}^{\prime}$ denotes the map $H^{\otimes i} \rightarrow H^{\otimes i+1}$ where $\Delta^{\prime}$ is applied on the first tensor factor only, and $m_{i}$ the map $H^{\otimes(i+1)} \rightarrow H^{\otimes i}$, where $m$ is applied on the first two tensor factors only, then we can rewrite (4) as

$$
(u \varepsilon-\mathrm{id})^{* k+1}=(-1)^{k+1} m m_{2} \cdots m_{k} \Delta_{k}^{\prime} \cdots \Delta_{2}^{\prime} \Delta^{\prime} .
$$

By splitting the powers in the form $\sigma^{*(k+1)}=\sigma * \sigma^{k}$, one can obtain a twin formula of (4), on which the coproduct is applied successively on the last tensor factor, instead of on the first factor; that formula was given in Ref. 1. 
While all the foregoing is happily elementary, the following important fact must be registered: a new grading on $H$ has been obtained, defined simply by declaring the degree of a generator $a$ as $k$ when $\sigma^{* k}(a) \neq 0$ and $\sigma^{* k+1}(a)=0$. It is easily seen that this indeed defines a grading $\delta$; the degree of a product is the sum of the degrees of the factors.

For a connected diagram $\Gamma \in \mathcal{H}$ this grading coincides with the maximal length of a chain of subdivergences inside $\Gamma$ (see below), and in this context we call it depth. We can combine $\delta$ with the gradings $\#$ or $\ell$ to obtain a bidegree: note that we have proved $\delta(\Gamma) \leq \#(\Gamma)$, for any $\Gamma$.

This grading by depth is the same $k$-primitivity grading already pondered in the seminal paper ${ }^{12}$ and studied in the context of $H_{R}$ by Broadhurst and Kreimer. ${ }^{13}$ Here the concept is even more pertinent, as the correlation between loop number and depth in field theory is weaker than the correlation between the number of tree vertices and $k$-primitivity in $H_{R}$ : for instance, it is well known that in the $\varphi_{4}^{4}$ model there are three 5-loop diagrams which are (1-)primitive. ${ }^{14}$

We write $\mathcal{H}^{(k)}$ for the space of elements of primitivity degree $k$.

Anticipating the following discussion, note that there are other ways to show that a connected graded bialgebra is a Hopf algebra. One can take advantage of the equation $m(S \otimes$ id $) \Delta(a)=0$ whenever $a \in H^{(n)}$ for $n \geq 1$, to introduce in the context the Bogoliubov recursive formula:

$$
S_{B}(a):=-a-\sum_{j} S_{B}\left(a_{j}^{\prime}\right) a_{j}^{\prime \prime}
$$

if $\Delta^{\prime} a=\sum_{j} a_{j}^{\prime} \otimes a_{j}^{\prime \prime}$.

Proposition 3. If $H$ is a connected, graded bialgebra, then $S(a)=S_{B}(a)$.

Proof. The statement holds, by a direct check, if $a \in H^{(1)}$. Assume that $S(b)=S_{B}(b)$ whenever $b \in H^{(k)}$ with $k \leq n$, and let $a \in H^{(n+1)}$. Then

$$
\begin{aligned}
S(a) & =\sigma(a)+\sum_{i=1}^{n} \sigma^{* i} * \sigma(a)=-a+m\left(\sum_{i=1}^{n} \sigma^{* i} \otimes \sigma\right) \Delta(a) \\
& =-a+m \sum_{i=1}^{n} \sigma^{* i} \otimes \sigma\left(a \otimes 1+1 \otimes a+\Delta^{\prime} a\right) \\
& =-a+\sum_{j} \sum_{i=1}^{n} \sigma^{* i}\left(a_{j}^{\prime}\right) \sigma\left(a_{j}^{\prime \prime}\right)=-a-\sum_{j} \sum_{i=1}^{n} \sigma^{* i}\left(a_{j}^{\prime}\right) a_{j}^{\prime \prime} \\
& =-a-\sum_{j} S_{B}\left(a_{j}^{\prime}\right) a_{j}^{\prime \prime}=S_{B}(a),
\end{aligned}
$$

where the penultimate equality uses the inductive hypothesis.

Taking into account that we can also write $S(a)=\sigma(a)+\sum_{i=1}^{n} \sigma * \sigma^{* i}(a)$, it follows that the twin formula

$$
S_{B}^{\prime}(a):=-a-\sum_{j} a_{j}^{\prime} S_{B}^{\prime}\left(a_{j}^{\prime \prime}\right)
$$

also provides an expression for the antipode. 
We record (6) in the language of the bialgebra $\mathcal{H}$ of graphs

$$
S(\Gamma):=-\Gamma-\sum_{\emptyset \nsubseteq \gamma \mp \Gamma} S(\gamma) \Gamma / \gamma
$$

For a primitive diagram, $S(\Gamma)=-\Gamma$.

Now it is time to reveal our strategy. Perhaps the main path-breaking insight of Ref. 12 and subsequent papers by Kreimer and coworkers is the introduction of the "twisted antipode". Let us usher in the other personages of this drama. There is a linear map $T: V \rightarrow V$, which effects the subtraction of ultraviolet divergencies in each renormalization scheme.

The twisted (or "renormalized") antipode $S_{T, f}$ is a map $\mathcal{H} \rightarrow V$ defined by $S_{T, f}(\emptyset)=1$; $S_{T, f}=T \circ f \circ S$ for primitive diagrams, and then recursively:

$$
S_{T, f} \Gamma=-[T \circ f] \Gamma-T\left[\sum_{\emptyset \varsubsetneqq \gamma \varsubsetneqq \Gamma} S_{T, f}(\gamma) f(\Gamma / \gamma)\right] .
$$

In other words, $S_{T, f}$ is the map that produces the counterterms in perturbative field theory. The Hopf algebra approach works most effectively because in many cases $S_{T, f}$ is multiplicative; for that, it is not necessary for $T$ to be an endomorphism of the algebra of amplitudes $V$, but the following weaker condition ${ }^{5,15,16}$ is sufficient:

$$
T(h g)=T(T(h) g)+T(h T(g))-T(h) T(g) .
$$

This condition endows $\mathcal{H}$ with the structure of a Rota-Baxter algebra (see Refs. 17,18); it is fulfilled in the BPHZ formalism and the dimensional regularization scheme with minimal subtraction, for which the present paradigm is most cleanly formulated. ${ }^{19}$

Finally, the renormalization map $R_{T, f}$ is given by

$$
R_{T, f}:=S_{T, f} * f .
$$

In view of a previous remark, $R_{T, f}$ is also a homomorphism; compatibility with the coproduct operation is given by its very definition as a convolution.

(In Epstein-Glaser renormalization, things are a bit more complicated, since $S_{T, f}$ is not properly defined, and $R_{T, f}$ involves a map between two different spaces of Feynman amplitudes; still, the homomorphism condition for $R_{T, f}$ can be enforced, and $R_{T, f}$ is compatible with the Hopf algebra structure in a suitable sense.)

In what follows, we shall assume that $S_{T, f}$ has been defined to be a homomorphism, and we concentrate on the computation of $S$. According to the dictum, Hopf algebras simplify combinatorics by reducing it to algebra. Connes and Kreimer's algebraic approach to the renormalization schemes separates neatly their combinatorics from the analytical procedures and renders the first an essentially trivial application of Hopf algebra.

Now, for the combinatorial aspect in renormalization theory, there are on the market mainly the recursive formula by Bogoliubov, Zimmermann's forest formula and the corrected Dyson-Salam formula. The last one is most natural in the context of the primitivity grading. They just amount to different ways to compute the antipode. It must be already evident that the recursive formula 
by Bogoliubov corresponds to the definition of $S_{B}$. Now, in order to prove the equivalence of two combinatorial schemes, it is enough to prove that both yield the antipode, either directly, as in the proof of Proposition 3, or by using the uniqueness of the antipode. This we systematically proceed to do in the sequel. That the coming proofs are all short, utterly simple, or decidedly trivial, is our main point and asset.

It turns out, and this is perhaps the most illuminating result, that the Dyson-Salam scheme corresponds identically (i.e., without need of further cancellations) to the geometric series formula.

\section{Convolution and the Dyson-Salam formula}

The present framework applies to proper and improper graphs. For brevity, in what follows we concentrate on proper graphs.

Definition 1. A chain $\mathcal{C}$ of a proper, connected graph $\Gamma$ is a sequence $\emptyset \varsubsetneqq \gamma_{1} \varsubsetneqq \gamma_{2} \varsubsetneqq \cdots \varsubsetneqq \gamma_{k} \varsubsetneqq \Gamma$ of proper, divergent, not necessarily connected subgraphs of $\Gamma$. We denote by $C(\Gamma)$ the set of chains of $\Gamma$. The length of a chain $\mathcal{C}$ is the number $l(\mathcal{C})=k+1=:|\mathcal{C}|+1$, and we write $\Omega(\mathcal{C}):=\gamma_{1}\left(\gamma_{2} / \gamma_{1}\right) \ldots\left(\gamma_{k-1} / \gamma_{k}\right)\left(\Gamma / \gamma_{k}\right)$.

With this notation we can define the antipode as follows:

$$
S_{D S}(\Gamma):=\sum_{\mathcal{C} \in C(\Gamma)}(-1)^{l(\mathcal{C})} \Omega(\mathcal{C})
$$

This definition corresponds, on the one hand, to the correct version of the Dyson-Salam formula for renormalization. On the other hand, formula (8) is totally analogous to the explicit expression for the antipode given by Schmitt for his incidence Hopf algebras. ${ }^{20}$

Proposition 4. $S_{D S}$ so defined is an antipode for $\mathcal{H}$.

Proof. We prove that $S_{D S}$ is an inverse, under convolution, of id. By definition,

$$
\begin{aligned}
S_{D S} * \operatorname{id}(\Gamma) & =\sum_{\gamma \subseteq \Gamma} S_{D S}(\gamma) \Gamma / \gamma=S_{D S}(\Gamma)+\sum_{\emptyset \subseteq \gamma \varsubsetneqq \Gamma} S_{D S}(\gamma) \Gamma / \gamma \\
& =S_{D S}(\Gamma)+\sum_{\emptyset \subseteq \gamma \varsubsetneqq \Gamma \mathcal{D} \in C(\gamma)} \sum_{(-1)^{l(\mathcal{D})} \Omega(\mathcal{D}) \Gamma / \gamma .}
\end{aligned}
$$

Now, if $\mathcal{D} \in C(\gamma)$, say $\mathcal{D}=\left\{\gamma_{1}, \ldots, \gamma_{k}\right\}$, then $\mathcal{C}=\left\{\gamma_{1}, \ldots, \gamma_{k}, \gamma\right\} \in C(\Gamma)$. Moreover,

$$
\Omega(\mathcal{C})=\Omega(\mathcal{D}) \Gamma / \gamma, \quad \text { and } \quad l(\mathcal{C})=l(\mathcal{D})+1
$$

On the other hand, given a chain $\mathcal{C}=\left\{\gamma_{1}, \ldots, \gamma_{n}\right\} \in C(\Gamma)$, then $\mathcal{D}=\left\{\gamma_{1}, \ldots, \gamma_{n-1}\right\} \in C\left(\gamma_{n}\right)$, and (9) holds. Therefore

$$
S_{D S} * \operatorname{id}(\Gamma)=S_{D S}(\Gamma)-\sum_{\mathcal{C} \in C(\Gamma)}(-1)^{l(\mathcal{C})} \Omega(\mathcal{C})=0=u \varepsilon(\Gamma)
$$

in other words, $S_{D S}$ is a left inverse for id, and therefore it is an antipode. 
As a corollary $S=S_{B}=S_{D S}$. Nevertheless, it is more instructive to check that $S=S_{D S}$ identically, as follows.

Proposition 5. $S_{D S}$ coincides with $S$ without cancellations.

Proof. First, given a proper, connected graph $\Gamma$, we rewrite

$$
S_{D S}(\Gamma):=\sum_{k}(-1)^{k+1} \sum_{\mathcal{C} \in C_{k}(\Gamma)} \Omega(\mathcal{C}),
$$

where $C_{k}(\Gamma)$ denote the set of chains of length $k+1$. Thus, it is enough to prove that

$$
(-1)^{k+1} \sum_{\mathcal{C} \in C_{k}(\Gamma)} \Omega(\mathcal{C})=(u \varepsilon-\mathrm{id})^{*(k+1)}(\Gamma)=\sigma^{*(k+1)}(\Gamma) .
$$

To prove this first we notice that

$$
\sum_{\emptyset \nsubseteq \gamma_{1} \subsetneq \gamma_{2} \cdots \subsetneq \gamma_{k}} \gamma_{1} \otimes \gamma_{2} / \gamma_{1} \otimes \cdots \otimes \gamma_{k} / \gamma_{k-1} \otimes \Gamma / \gamma_{k}=\Delta_{k}^{\prime} \cdots \Delta_{2}^{\prime} \Delta^{\prime}(\Gamma) .
$$

Indeed, by definition of the coproduct the statement is true for $k=1$. Moreover, if (10) holds for $k-1$, then

$$
\begin{aligned}
& \sum_{\emptyset \nsubseteq \gamma_{1} \varsubsetneqq \gamma_{2} \cdots \varsubsetneqq \gamma_{k} \subsetneq \Gamma} \gamma_{1} \otimes \gamma_{2} / \gamma_{1} \otimes \cdots \otimes \gamma_{k} / \gamma_{k-1} \otimes \Gamma / \gamma_{k} \\
& =\sum_{\emptyset \nsubseteq \gamma_{2} \subsetneq \gamma_{3} \cdots \varsubsetneqq \gamma_{k} \subsetneq \Gamma} \Delta_{k}^{\prime}\left(\gamma_{2} \otimes \gamma_{3} / \gamma_{2} \otimes \cdots \otimes \gamma_{k} / \gamma_{k-1} \otimes \Gamma / \gamma_{k}\right) \\
& =\Delta_{k}^{\prime}\left(\sum_{\emptyset \varsubsetneqq \gamma_{1} \varsubsetneqq \gamma_{2} \cdots \varsubsetneqq \gamma_{k-1} \subsetneq \Gamma} \gamma_{1} \otimes \gamma_{2} / \gamma_{1} \otimes \cdots \otimes \gamma_{k-1} / \gamma_{k-2} \otimes \Gamma / \gamma_{k-1}\right) \\
& =\Delta_{k}^{\prime} \Delta_{k-1}^{\prime} \cdots \Delta_{2}^{\prime} \Delta^{\prime}(\Gamma) \text {. }
\end{aligned}
$$

Thus, by (5)

$$
\begin{aligned}
(-1)^{k+1} \sum_{\mathcal{C} \in C_{k}(\Gamma)} \Omega(\mathcal{C}) & =(-1)^{k+1} \sum_{\emptyset \varsubsetneqq \gamma_{1} \subsetneq \gamma_{2} \cdots \varsubsetneqq \gamma_{k} \subsetneq \Gamma} \gamma_{1}\left(\gamma_{2} / \gamma_{1}\right) \cdots\left(\gamma_{k} / \gamma_{k-1}\right)\left(\Gamma / \gamma_{k}\right) \\
& =(-1)^{k+1} m m_{2} \cdots m_{k} \Delta_{k}^{\prime} \cdots \Delta_{2}^{\prime} \Delta^{\prime}(\Gamma)=\sigma^{*(k+1)}(\Gamma) .
\end{aligned}
$$

The proof shows how the chains are generated from the coproduct.

As an application, we obtain a nonrecursive formula for $S_{T, f}$ : if $\mathcal{C}=\left\{\gamma_{1}, \ldots, \gamma_{k}\right\}$ is a chain in $C(\Gamma)$, write

$$
\Omega_{T, f}(\mathcal{C}):=T\left(T\left[\cdots T\left(T\left[T\left(f\left(\gamma_{1}\right)\right) f\left(\gamma_{1} / \gamma_{2}\right)\right] f\left(\gamma_{2} / \gamma_{3}\right)\right) \cdots f\left(\gamma_{k-1} / \gamma_{k}\right)\right] f\left(\Gamma / \gamma_{k}\right)\right) .
$$

Let us use the temporary notation

$$
\tilde{S}_{T, f} \Gamma:=\sum_{\mathcal{C} \in C(\Gamma)}(-1)^{l(\mathcal{C})} \Omega_{T, f}(\mathcal{C})=\sum_{k=0}^{\delta(\Gamma)}(-1)^{k+1} \sum_{\mathcal{C} \in C_{k}(\Gamma)} \Omega_{T, f}(\mathcal{C}) .
$$


Proposition 6. $S_{T, f}=\tilde{S}_{T, f}$.

Proof. We shall proceed by induction on the bidegree (no other method seems available here). A simple check gives the statement for $\Gamma \in \mathcal{H}^{(1)}$. Assume the claim is true for graphs in $\mathcal{H}^{(l)}$ with $l \leq k$, and let $\Gamma \in \mathcal{H}^{(k+1)}$, then

$$
\begin{aligned}
S_{T, f} \Gamma & =-[T \circ f] \Gamma-T\left[\sum_{\emptyset \varsubsetneqq \gamma \mp \Gamma} S_{T, f}(\gamma) f(\Gamma / \gamma)\right] \\
& =-[T \circ f] \Gamma-T\left[\sum_{\emptyset \nsubseteq \gamma \mp \Gamma \mathcal{D} \in C(\gamma)} \sum_{\left.(-1)^{l(\mathcal{D})} \Omega_{T, f}(\mathcal{D}) f(\Gamma / \gamma)\right],}\right.
\end{aligned}
$$

since each $\gamma \in \mathcal{H}^{(l)}$ for some $l \leq k$. Now, if $\mathcal{D} \in C(\gamma)$, then $\mathcal{C}=\mathcal{D} \cup\{\gamma\} \in C(\Gamma)$,

$$
l(\mathcal{C})=l(\mathcal{D})+1, \quad \text { and } \quad \Omega_{T, f}(\mathcal{C})=T\left[\Omega_{T, f}(\mathcal{D}) f(\Gamma / \gamma)\right] .
$$

Conversely, if $\mathcal{C}=\left\{\gamma_{1}, \ldots, \gamma_{n}\right\} \in C(\Gamma)$ is not the trivial chain $\{\emptyset\}$, then $\mathcal{D}=\left\{\gamma_{1}, \ldots, \gamma_{n-1}\right\}$ is a chain in $C\left(\gamma_{n}\right)$, and

$$
(-1)^{l(\mathcal{C})} \Omega_{T, f}(\mathcal{C})=-T\left[(-1)^{l(\mathcal{D})} \Omega_{T, f}(\mathcal{D}) f\left(\Gamma / \gamma_{n}\right)\right] .
$$

Therefore

$$
S_{T, f}=\sum_{\mathcal{C} \in C(\Gamma)}(-1)^{l(\mathcal{C})} \Omega_{T, f}(\mathcal{C})=\tilde{S}_{T, f}
$$

The morals of the story so far are: first, there is nothing in Bogoliubov's procedure that will not be valid in any connected, graded bialgebra; second, Schmitt's formula for his incidence Hopf algebras coincides identically with the geometric series formulae (3) and (4); third, the latter in the field theory context gives rise to the Dyson-Salam formula. We turn our attention now to Zimmermann's forest formula.

\section{Zimmermann's forest formula}

Definition 2. A (normal) forest $\mathcal{F}$ of a proper, connected graph $\Gamma$ is a set of proper, divergent and connected subdiagrams, none of them equal to $\Gamma$, such that any pair of elements are nonoverlapping. Again we include the forest $\{\emptyset\}$ as a special case. $F(\Gamma)$ denotes the set of forests of $\Gamma$. The density of a forest $\mathcal{F}$ is the number $d(\mathcal{F})=|\mathcal{F}|+1$, where $|\mathcal{F}|$ is the number of elements of $\mathcal{F}$. Given $\gamma \in \mathcal{F}$ we say that $\gamma^{\prime}$ is a predecessor of $\gamma$ in $\mathcal{F}$ if $\gamma^{\prime} \varsubsetneqq \gamma$ and there is no element $\gamma^{\prime \prime}$ in $\mathcal{F}$ such that $\gamma^{\prime} \varsubsetneqq \gamma^{\prime \prime} \varsubsetneqq \gamma$. Let

$$
\Theta(\mathcal{F}):=\prod_{\gamma \in \mathcal{F} \cup\{\Gamma\}} \gamma / \tilde{\gamma}
$$

where $\tilde{\gamma}$ denote the disjoint union of all predecessors of $\gamma$. When $\gamma$ is minimal, $\tilde{\gamma}=\emptyset$, and $\gamma / \tilde{\gamma}=\gamma$. 


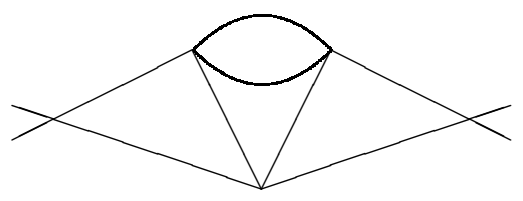

Figure 2: Diagram $\Gamma$ without extra cancellations in $S_{Z}(\Gamma)$ with respect to $S_{D S}(\Gamma)$

Notice that if a forest $\mathcal{F}$ is a chain, then $\Theta(\mathcal{F})=\Omega(\mathcal{F})$, and conversely if a chain $\mathcal{C}$ is a forest, $\Omega(\mathcal{C})=\Theta(\mathcal{C})$. Obviously not every forest is a chain; but also not every chain is a forest, because product subgraphs can occur in chains and cannot in forests. There are diagrams like the one in the $\varphi_{4}^{4}$ model pictured in Figure 2 , for which the sets of chains and forests coincide; but in general there are fewer forests than chains.

Zimmermann's version for the antipode is defined by

$$
S_{Z}(\Gamma):=\sum_{\mathcal{F} \in F(\Gamma)}(-1)^{d(\mathcal{F})} \Theta(\mathcal{F}) .
$$

Proposition 7. $S_{Z}$ provides another formula for the antipode of $\mathcal{H}$.

Proof. Once more, the idea is to prove that $S_{Z}$ is an inverse, under convolution, of id. By definition

$$
\begin{aligned}
S_{Z} * \operatorname{id}(\Gamma) & =\sum_{\gamma \subseteq \Gamma} S_{Z}(\gamma) \Gamma / \gamma=S_{Z}(\Gamma)+\sum_{\emptyset \subseteq \gamma \mp \Gamma} S_{Z}(\gamma) \Gamma / \gamma \\
& =S_{Z}(\Gamma)+\sum_{\emptyset \subseteq \gamma \subsetneq \Gamma}\left(\prod_{i=1}^{n_{\gamma}} S_{Z}\left(\lambda_{i}^{\gamma}\right)\right) \Gamma / \gamma \\
& =S_{Z}(\Gamma)+\sum_{\emptyset \subseteq \gamma \varsubsetneqq \Gamma} \prod_{i=1}^{n_{\gamma}}\left(\sum_{\mathcal{F}_{i} \in F\left(\lambda_{i}^{\gamma}\right)}(-1)^{d\left(\mathcal{F}_{i}\right)} \Theta\left(\mathcal{F}_{i}\right)\right) \Gamma / \gamma
\end{aligned}
$$

where $n_{\gamma}$ is the number of connected components of $\gamma$, and $\lambda_{i}^{\gamma}, i=1, \ldots, n_{\gamma}$ are the connected components of $\gamma$.

Now, if $\mathcal{F}_{i} \in F\left(\lambda_{i}^{\gamma}\right)$, then $\mathcal{F}:=\bigcup_{i=1}^{n_{\gamma}}\left(\mathcal{F}_{i} \cup\left\{\lambda_{i}^{\gamma}\right\}\right)$ is a forest of $\Gamma$. Moreover

$$
|\mathcal{F}|=\sum_{i=1}^{n_{\gamma}}\left|\mathcal{F}_{i}\right|+n_{\gamma}=\sum_{i=1}^{n_{\gamma}} d\left(\mathcal{F}_{i}\right),
$$

so

$$
d(\mathcal{F})=\sum_{i=1}^{n_{\gamma}} d\left(\mathcal{F}_{i}\right)+1
$$

On the other hand, in $\mathcal{F}, \Gamma / \tilde{\Gamma}=\Gamma / \gamma$, hence

$$
\Theta(\mathcal{F})=\left(\prod_{i=1}^{n_{\gamma}} \Theta\left(\mathcal{F}_{i}\right)\right) \Gamma / \tilde{\Gamma}=\left(\prod_{i=1}^{n_{\gamma}} \Theta\left(\mathcal{F}_{i}\right)\right) \Gamma / \gamma
$$


Conversely, if $\mathcal{F} \in F(\Gamma)$, and if $\gamma_{1}, \ldots, \gamma_{k}$ are the maximal elements of $\mathcal{F}$, then the sets $\mathcal{F}_{i}:=$ $\left\{\gamma \in \mathcal{F}: \gamma \varsubsetneqq \gamma_{i}\right\}$ constitute a forest of $\gamma_{i}$. Since all the elements of a forest are connected diagrams, $\gamma_{1}, \ldots, \gamma_{k}$ are the connected components of $\gamma=\prod_{i=1}^{k} \gamma_{i}$ and clearly (11) and (12) hold. Therefore,

$$
S_{Z} * \operatorname{id}(\Gamma)=S_{Z}(\Gamma)-\sum_{\mathcal{F} \in F(\Gamma)}(-1)^{d(\mathcal{F})} \Theta(\mathcal{F})=0=u \varepsilon(\Gamma) .
$$

Thus, $S_{Z}$ is a left inverse for id, and therefore is an antipode.

The proofs of Propositions 4 and 7 are parallel; the difference lies in minor combinatorial details. Even so, it is clear that Zimmermann's formula (although more sensitive in practice to the details of the renormalization method) is, from the combinatorial viewpoint, altogether subtler than Bogoliubov's or Dyson and Salam's. It is more economical in that all the cancellations implicit in the convolution formula (3) are taken into account and suppressed; this we already made clear in the context of the algebra of rooted trees. ${ }^{1}$ Thus, the "commerce" between quantum field theory and Hopf algebra theory has not been one-way: Zimmermann's formula is advantageoulsy applicable to a large class of bialgebras.

The reader will have no difficulty in writing the nonrecursive forest formula for the twisted coinverse $S_{T, f}$.

\section{The bidegree and computations in quantum field theory}

Let us indicate first that Kreimer has announced ${ }^{17}$ a new proof of finiteness of the renormalized graphs and Green functions, based on a cohomological reinterpretation of the basic coproduct equation (11).

Whether the Connes-Kreimer algebraic paradigm will become useful to simplify computations —in distinction to "merely" proofs — in realistic field theories, seems to hinge to a large extent on the practical usefulness of the depth bigrading. The fact is that simplifications in sums of Feynman diagrams do occur, and they usually involve, beyond trivialization of the topology, reduction in depth. For instance, in the $\varphi_{4}^{4}$ model we have (for the corresponding amplitudes in configuration space) the situation described in Figure 3 .

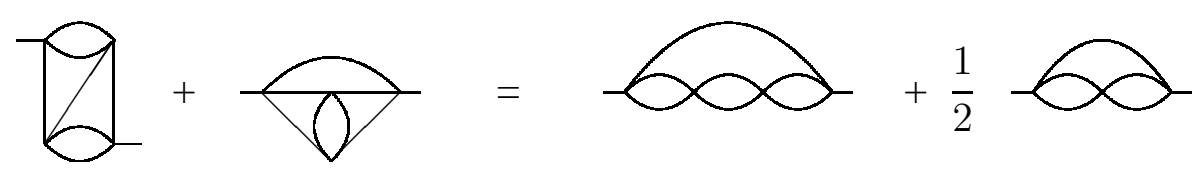

Figure 3: $\zeta(3)$ coefficients vanish in the sum of two graphs with the same symmetry factor

A naive hope in that respect, to wit, that every diagram be eventually expressed in terms of primitive elements (so renormalization proceeds "at a stroke") is quickly dashed. A Hopf algebra $H$ is primitively generated when the smallest subalgebra of $H$ containing all its primitive elements is $H$ itself. The structure theorem for commutative connected graded algebras ${ }^{21}$ makes it plain that Hopf algebras of Feynman graphs are far from being primitively generated; neither the Hopf 
algebra of rooted trees nor its noncommutative geometry subalgebra $H_{\mathrm{CM}}$ (Ref. 22) are primitively generated.

In fact, only elements for which the coproduct is invariant under the flip map $a \otimes b \mapsto b \otimes a$ can be primitively generated. In any Hopf algebra associated to a field theory there exist "ladder" subalgebras of diagrams with only completely nested subgraphs, and these subalgebras are primitively generated. However, this is of scant practical use, as then the recursive methods ${ }^{23}$ carry off the award for computational simplicity.

According to the structure theorem, commutative Hopf algebras can be decomposed as algebras as a tensor product

$$
H=S(P(H)) \otimes S\left(W_{H}\right),
$$

where $S(P(H)$ ) denotes the polynomial algebra generated by all the primitive elements in $H$, and $S\left(W_{H}\right)$ the polynomial algebra on a (nonunique) suitable subspace $W_{H}$ of $H$. To get a handle on (a representative for) $W_{H}$ for bialgebras of Feynman graphs is on the order of the day.

The depth grading for the algebra $H_{R}$ of rooted trees has been investigated, beyond Ref. 13, in Ref. 24. The strategy suggested in Ref. 24 looks feasible in bialgebras of graphs. By use of the dual algebra, so-called normal coordinate elements (appropriate sums of products of graphs) can be found, for which the antipode (although not the twisted antipode in general) is diagonal.

Let $Z_{\gamma}$ be the dual element of a graph $\gamma$. Any graph $\Gamma$ has an associated normal element $\psi_{\Gamma}$, and any graph can be decomposed into a sum of products of normal elements. Let a sequence of graphs $J=\left(\gamma_{1}, \ldots, \gamma_{k}\right)$ be given; we say that $\Gamma$ is compatible with $J$ if

$$
\left\langle Z_{\gamma_{1}} \otimes \ldots \otimes Z_{\gamma_{k}}, \Delta^{k-1} \Gamma\right\rangle \neq 0
$$

The normal decomposition is as follows:

$$
\Gamma=\psi_{\Gamma}+\sum_{k \geq 2} \frac{\left\langle Z_{\gamma_{1}} \otimes \ldots \otimes Z_{\gamma_{k}}, \Delta^{k-1} \Gamma\right\rangle}{k !} \psi_{\gamma_{1}} \ldots \psi_{\gamma_{k}}
$$

where we sum in practice over a finite number of compatible sequences.

Ladder normal coordinate elements are primitive, and for non-ladder ones indications are that the complexity of their renormalization is substantially lessened with respect to that of the graphs themselves; they are instrumental in the description of $W_{H}$.

Also, Kreimer has introduced ${ }^{17}$ a "shuffle" product of diagrams, based on a variant of (13), that seems to hold promise of eventual factorization of perturbative field theory into primitive elements. In this respect, as in other tantalizing subjects springing from the Connes-Kreimer paradigm, we are barely starting to scratch the surface.

\section{Acknowledgements}

We thank Joseph C. Várilly for illuminating discussions and $\mathrm{T}_{\mathrm{E}} \mathrm{Xnical}$ help. We are grateful for the hospitality of the Departamento de Física Teórica of the Universidad de Zaragoza. Support from the Vicerrectoría de Investigación of the Universidad de Costa Rica is acknowledged. 


\section{A Appendix: Graded bialgebras}

A bialgebra $\mathrm{H}$ is a vector space over a field $\mathbb{F}$ (here taken to be of characteristic 0) equipped with two structures: an algebra structure and a coalgebra structure, related by some compatibility conditions. The algebra structure is described by two maps: the product $m: H \otimes H \rightarrow H$, and the unit map $u: \mathbb{F} \rightarrow H$. The conditions imposed on these maps are:

1. Associativity: $m(m \otimes \mathrm{id})=m(\mathrm{id} \otimes m): H \otimes H \otimes H \rightarrow H$;

2. Unity: $m(u \otimes \mathrm{id})=m(\mathrm{id} \otimes u)=\mathrm{id}: H \rightarrow H$.

A coalgebra is obtained by reversing arrows in the defining maps for an algebra; it is, therefore, also described by two maps: the coproduct $\Delta: H \rightarrow H \otimes H$, and the counit $\varepsilon: H \rightarrow \mathbb{F}$. The requirements are:

3. Coassociativity: $(\Delta \otimes \mathrm{id}) \Delta=(\mathrm{id} \otimes \Delta) \Delta: H \rightarrow H \otimes H \otimes H$;

4. Counity: $(\varepsilon \otimes \mathrm{id}) \Delta=(\mathrm{id} \otimes \varepsilon) \Delta=\mathrm{id}: H \rightarrow H$.

Finally, to obtain a bialgebra one stipulates

5. Compatibility: $\Delta$ and $\varepsilon$ are unital algebra homomorphisms.

This requirement turns out to be equivalent to asking that $m$ and $u$ be coalgebra morphisms.

Definition 3. A bialgebra $H=\bigoplus_{n=0}^{\infty} H^{(n)}$ graded as a vector space is called a graded bialgebra when the grading is compatible with both the algebra and the coalgebra structures:

$$
H^{(n)} H^{(m)} \subseteq H^{(n+m)} \quad \text { and } \quad \Delta\left(H^{(n)}\right) \subseteq \bigoplus_{p+q=n} H^{(p)} \otimes H^{(q)} .
$$

It is called connected when the first piece consists of scalars only: $H^{(0)}=\mathbb{F} 1$.

A most useful property of connected graded bialgebras is that when $a \in H^{(n)}$, the coproduct can be written as

$$
\Delta a=a \otimes 1+1 \otimes a+\sum_{j} a_{j}^{\prime} \otimes a_{j}^{\prime \prime},
$$

where the elements $a_{j}^{\prime}$ and $a_{j}^{\prime \prime}$ all have degree between 1 and $n-1$. The proof is easy and found in many places. ${ }^{11,15,25,26,27}$ To simplify the notation we define

$$
\Delta^{\prime} a:=\Delta a-a \otimes 1-1 \otimes a=\sum_{j} a_{j}^{\prime} \otimes a_{j}^{\prime \prime} .
$$

Coassociativity of $\Delta^{\prime}$ is easily obtained from the coassociativity of $\Delta$. An element $a \in H$ is called (1-)primitive when $\Delta^{\prime} a=0$.

Applying $\varepsilon \otimes$ id to (14a) gives $a=(\varepsilon \otimes \mathrm{id})(\Delta a)=\varepsilon(a) 1+a+\sum_{j} \varepsilon\left(a_{j}^{\prime}\right) a_{j}^{\prime \prime}$; therefore, if $a \in H^{(n)}$ with $n \geq 1$, the connectedness condition forces $\varepsilon(a)=0$. 


\section{References}

1. H. Figueroa and J. M. Gracia-Bondía, Mod. Phys. Lett. A16, 1427 (2001).

2. A. Connes and D. Kreimer, Commun. Math. Phys. 199, 203 (1998).

3. W. Zimmermann, Commun. Math. Phys. 15, 208 (1969).

4. E. B. Manoukian, Renormalization, (Academic Press, London, 1983).

5. A. Connes and D. Kreimer, Commun. Math. Phys. 210, 249 (2000).

6. J. M. Gracia-Bondía and S. Lazzarini, Connes-Kreimer-Epstein-Glaser renormalization, hep-th/0006106

7. J. M. Gracia-Bondía, Math. Phys. Analysis and Geometry 6, 59 (2003).

8. W. Zimmermann, Remarks on equivalent formulations for Bogoliubov's method of renormalization, in Renormalization Theory, eds. G. Velo and A. S. Wightman, NATO ASI Series C 23 (D. Reidel, Dordrecht, 1976).

9. D. Kastler, On the external structure of graphs, to appear in J. Math. Phys.

10. G. Pinter, Lett. Math. Phys. 54, 227 (2000).

11. J. M. Gracia-Bondía, J. C. Várilly and H. Figueroa, Elements of Noncommutative Geometry (Birkhäuser, Boston, 2001).

12. D. Kreimer, Adv. Theor. Math. Phys. 2, 303 (1998).

13. D. J. Broadhurst and D. Kreimer, Commun. Math. Phys. 215, 217 (2000).

14. H. Kleinert and V. Schulte-Frohlinde, Critical Properties of $\phi^{4}$ Theories (World Scientific, Singapore, 2001).

15. J. C. Várilly, Hopf algebras in noncommutative geometry, in Geometrical and Topological Methods in Quantum Field Theory, eds. A. Cardona, H. Ocampo and S. Paycha (World Scientific, Singapore, 2003).

16. D. Kreimer, Adv. Theor. Math. Phys. 3, 3 (1999).

17. D. Kreimer, Ann. Phys. 303, 179 (2003).

18. K. Ebrahimi-Fard, L. Guo and D. Kreimer, Integrable renormalization II: the general case, hep-th/0403118

19. A. Connes and D. Kreimer, Commun. Math. Phys. 216, 215 (2001).

20. W. R. Schmitt, J. Comb. Theory A46, 264 (1987); J. Pure Appl. Alg. 96, 299 (1994).

21. K.-H. Hoffmann and S. A. Morris, The Structure of Compact Groups (de Gruyter, Berlin, 1998).

22. A. Connes and H. Moscovici, Commun. Math. Phys. 198, 198 (1998).

23. I. Bierenbaum, Die Riemannsche $\zeta$-Funktion in iterierten Einschleifenintegralen (Diplomarbeit, Mainz, 2000).

24. C. Chryssomalakos, H. Quevedo, M. Rosenbaum and J. D. Vergara, Commun. Math. Phys. 225, 465 (2002).

25. N. Bourbaki, Groupes et Algèbres de Lie (Hermann, Paris, 1972).

26. D. Kastler, Connes-Moscovici-Kreimer Hopf algebras, in Mathematical Physics in Math-

ematics and Physics: Quantum and Operator Algebraic Aspects, ed. R. Longo, Fields Institute Communications 30 (American Mathematical Society, Providence, RI, 2001).

27. S. Montgomery, Hopf Algebras and their Actions on Rings, CBMS Regional Conference Series in Mathematics 82, (American Mathematical Society, Providence, RI, 1993). 